\title{
What remains of probability?
}

\author{
László E. Szabó \\ Department of Logic, Institute of Philosophy \\ Eötvös University, Budapest
}

1. When I say "probability" I do not mean a Kolmogorovian 'probability measure' or similar mathematical notion. Of course, a definition - both coordinative ${ }^{1}$ and logicalplays a constitutive role in the very concept to be defined. Nevertheless, there seems no reason to include into the definition of probability that it "satisfies the Kolmogorovian axioms of probability theory". For, once we know-supposedly from the rest part of the definition-what "probability" is in our world, it becomes a contingent fact of the world whether it satisfies the Kolmogorovian axioms or not; which can be known by a posteriori means. In other words, the aim of the so called "interpretations" of probability is not to find an interpretation of the Kolmogorovian axioms, but to give a sound meaning to scientific statements containing the term "probability".

For example, consider the following assertions of quantum mechanics or statistical mechanics:

$$
\begin{aligned}
p(a) & =\operatorname{tr}\left(\hat{P}_{a} \hat{W}\right) \\
p\left(\left\{N_{i}\right\}_{i=1,2, \ldots}\right) & =\frac{\left(\sum_{i} N_{i}\right) !}{\prod_{i} N_{i} !}
\end{aligned}
$$

And compare them with other similar scientific assertions, like the Coulomb law,

$$
\mathbf{E}(\mathbf{r})=q \frac{\mathbf{r}-\mathbf{r}_{q}}{\left|\mathbf{r}-\mathbf{r}_{q}\right|^{3}}
$$

or just a simple statement about the length of a rod:

$$
l=4 \mathrm{~m}
$$

In case (3) and (4) it is clear what the formulas assert. When we assert that the static electric field strength of a point charge is equal to $q \frac{\mathbf{r}-\mathbf{r}_{q}}{\left|\mathbf{r}-\mathbf{r}_{q}\right|^{3}}$, we have a previously defined 
physical quantity, electric field strength, and (3) expresses a contingent fact about this quantity.

By "definition" I mean empirical/operational/verificationist definition. This is not the place to argue for verificationism or operationalism. I only mention that my approach is based on a very weak operationalist/verificationist premise: scientific terms assigned to quantities like the ones appearing in (1)-(4) must have empirical definitions (except if the equations in question were definitions). In other words, those sentences of a scientific theory which are supposed to describe objective facts of the world must be expressible in observational/operational terms. Without this condition a scientific theory could not be empirically confirmable or disconfirmable. I believe, this view is widely accepted among physicists; although, the precise operational definition of a physical quantity can be a non-trivial issue, even in the case of basic spatiotemporal quantities. $^{2}$ However, in itself, this premise is not yet equivalent to operationalism or verificationism in general philosophical sense. It does not generally imply that a statement is necessarily meaningless if it is neither analytic nor empirically verifiable.

Now, contrary to (3) and (4), it is far from obvious what formulas (1) and (2) actually assert. What is the definition of the quantities on the left hand side of these formulas? What is the probability of an event? This is the basic question of the philosophy of probability. Strangely enough, in spite of the fact that the term "probability" is used in the everyday scientific discourse, there is a consensus in the philosophical literature $^{34}$ that we have no satisfactory answer to this question.

2. The various approaches can be divided into two major groups. According to the objectivist school, the probability of an event is something which characterizes a feature of the external world; roughly speaking, it is a property of the event and the circumstances. According to the subjectivist approach, on the contrary, the probability of an event is something which characterizes a feature of the internal world; it is not a property of the event and the circumstances, but a property of a particular intentional state of mind about the event and the circumstances; a "degree" of belief. Objectivists' probability is often called "chance" $(c h)$; subjectivists' probability is called "subjective probability" or "credence" ( $c r)$. Thus, it must be emphasized that chance and credence are not different interpretations of the same thing, but they are two different things, belonging to different types of phenomena. If they exist in our world, they do so independently; if they are at all connected, their connection must be a contingent fact of the world. Nevertheless, we still don't know exactly what chance is and what credence is.

3. No doubt, there is such a thing as a person's credence or belief; and, no doubt, it is meaningful to talk about the degree of a belief, as a belief can be stronger or weaker; and perhaps one can characterize it with a number between 0 and 1 , just like a numeric scale from 0 to 10 can be asked to communicate the intensity of a patient's pain. And one can easily imagine some rules governing the complex mental processes that determine this number; a dynamics by which this number changes in time under various conditions.

All these things belong to the scope of ordinary empirical sciences like psychology, cognitive science, human ethology, or sociology. Strangely enough, however, in the 
subjectivist literature, we cannot find any reference to the results of these empirical sciences. It worth mentioning that the assertions in question are, at the same time, quite ambitious. Consider only two examples.

Lewis' Principal Principle asserts that a person's credence is strictly determined by some other mental states, namely:

$$
\operatorname{cr}\left(\left.A\right|^{`} \operatorname{ch}(A)=x^{\prime} \& K\right)=x
$$

where ' $\operatorname{ch}(A)=x$ ' stands for the person's knowledge that $\operatorname{ch}(A)=x$ and $K$ stands for some further "admissible" (mis)information. If this is true, it is quite a strict causal/dynamical law of mental processes. Not to mention that those conditions that make proposition ' $c h(A)=x$ ' knowledge (that is, true) and proposition $K$ admissible, are conditions in the external world. So, in its stronger understanding, the principle is a statement about the relationship between the external world and one's mental states.

My second example is the Bayesian law of confirmation. It describes how the degree of a person's belief in the truth of $A$ changes due to getting information about a new evidence $E$ :

$$
c r_{t_{2}}(A)=c r_{t_{1}}(A \mid E)=c r_{t_{1}}(A) \frac{c r_{t_{1}}(E \mid A)}{c r_{t_{1}}(E)}
$$

where $c h_{t_{1}}$ is the person's previous credence function based on some earlier body of believes, $c h_{t_{2}}$ is the new credence function, based on the previous believes plus $E$. This, too, is a very strong claim about the dynamics of mental processes.

4. How is it that the subjectivist interpretation of probability can claim so precise quantitative laws about human mind without any reference to the results of empirical sciences? There are two standard explanations of this ignorance, and both raise further problems.

The first possibility is that the whole subjectivist theory is regarded as a kind of "armchair metaphysics". Typically, in Lewis' "Subjectivist's guide" the Principal Principle is based on some "evidences" drawn from his own intuitive answers to his Questionnaire. He writes:

I have given undefended answers to my four questions. I hope you found them obviously right, so that you will be willing to take them as evidence for what follows. If not, do please reconsider. If so, splendid-now read on. 5

That is, we make a priori assertions about the real world, on the basis of our everyday pre-scientific and pre-philosophical intuitions, without any reference to the epistemic means by which the asserted facts of the world can be accessed.

However, if a statement is obviously true in our world, then the statement must have an obvious meaning; there must be an obvious way in which the statement can be verified, whether it is true indeed, or not. In other words, we need some empirical/coordinative definitions of the basic terms "credence" and "chance".

The second possibility is that the subjectivist theory is regarded not as a theory about real persons' believes, but about the "credences of an abstract agent". Terms 
like "chance", "credence" or "agent" are only mathematical terms without any reference to the real world; the whole theory is a formal/mathematical construction, just like Kolmogorov's axiomatic theory of probability, group theory, or geometry. In this mathematical construction, one can define a "gamble" in which the agent is making "bets" on "outcome events"; one can define the notion of "rational agent " as an agent whose bets and credences are in a certain relation. One may make assumptions about the "behavior of the gambling device"; for example one can assume that the "chances of the outcome events" satisfy the axioms of Kolmogorov's probability theory; and from these premises, for example, one can prove the Dutch book theorem.

This is, of course, possible, but I believe this is not the final aim of subjectivist interpretation of probability. At the end of the day we would like to apply these abstract constructions in the metaphysical, epistemological, and scientific discourse about the real world; therefore we need to know how to apply our theoretical terms like "credence" and "chance". We would need to understand the meaning of these terms even if the rules of subjectivist theory of probability should not be seen as a factual description of actual human reasoning, but rather as a "normative standard of rationality"; since we need to understand the rules in order to follow them.

Thus, in either case, what we are missing is the empirical/operational/verificationist definitions of the basic concepts, first of all of "credence"; that is to say, a subjectivist's guide to objective chance would, first of all, require a guide to the subjectivist's credence.

5. Therefore, it could only lead to circularities if the definition of chance $\operatorname{ch}(A)$ included any essential reference to ' $\operatorname{ch}(A)=x$ ' in the Principal Principle. The reason is that the alleged relation (5) holds only if proposition $K$ is "admissible". "Admissibility" is, however, a concept the definition of which requires prior definitions of credence and chance, independently:

A proposition $P$ is admissible with respect to an outcome-specifying proposition $E$ for chance set-up $S$ ( $E$ says that event $e$ occurs) iff $P$ contains only the sort of information whose impact on reasonable credence about $E$, if any, comes entirely by way of impact on credence about the chances of those outcomes. [my italics] ${ }^{6}$

6. Let us return to the original problem of what "probability" means in the probabilistic assertions of the sciences. First, it is worth pointing out some conceptual confusion which needs to be sorted out. It is obvious that the concept of probability in science, especially in physics, is objective probability. When the behavior of a physical system is described by means of a probabilistic model, probabilities are supposed to describe some objective features of the external world; no matter if the underlying physical processes are deterministic or indeterministic. Classical statistical mechanics is a typical example. We believe that the detailed process is governed by the deterministic laws of classical mechanics; but, because of lacking the knowledge of the details, we give a less detailed probabilistic model. The probabilities in such a model are sometimes called "epistemic" probabilities. This is however a misleading terminology of the physicists, which differs from the terminology of the philosophers. For, the reason why we give a 
probabilistic description of the system, instead of a completely detailed deterministic one, is indeed related with a lack of knowledge, yet the probabilities in the model are objective probabilities and have nothing to do with "knowledge", "lack of information", etc. All statements of the probabilistic description of the system remain valid even if we get complete information about the details; because none of the objective features of the system described by the probabilities in question changes by knowing more information about the system.

Let me give an everyday example. You are waiting for the next train in a subway station. If you knew the exact timetable, you could make predictions like "The next train will arrive in 3 minutes." If you don't know the timetable but only know that the trains come in every 5 minutes, you can make less ambitious claims. For example, you can say that "I will wait less than 5 minutes."; or you can predict the following result of a long-run experiment: "Providing that the moments at which I enter to the station are uniformly distributed in time, the long-run average of my waiting time is 2.5 minutes." Now, the validity of these claims does not change if you get know the timetable.

7. What we observe here is nothing but a kind of Humean supervenience. Objective probabilities supervene on the collection of the particular facts of the actual history of the world, that is, all occurrent facts in all regions of spacetime; on the Humean Mosaic. And this is true, no matter if the world is deterministic or indeterministic; either in the sense that the different time slices of the actual history are not functionally related; either in the sense that there exist other possible histories of the world besides the actual one; either in the sense of a more sophisticated branching structure of possible spacetime-histories.

On the one hand this is trivially true; on the other hand, one has to put it in a more precise form: The truth or falsity of all meaningful statements about objective probabilities supervene on the Humean Mosaic, where "meaningful" is meant in a verificationist sense; that is, a statement is meaningful if it is expressible in terms of the Humean Mosaic.

Note, however, that this is also true for subjective probabilities: The truth or falsity of all meaningful statements about subjective probabilities supervene on the Humean Mosaic. The question is, of course: what are the meaningful statements about subjective probabilities? Another note: although the truth or falsity of all meaningful statements about both objective and subjective probabilities are determined by the actual content of Humean Mosaic, their truth or falsity can be known only by a posteriori means.

8. Nevertheless, what is chance and what is credence? We still do not have a tenable definition of probability, neither objective nor subjective. And how is it possible that physics and other empirical sciences do not notice problems arising from these unanswered fundamental questions? As to the concept of objective probability, in one of my earlier papers ${ }^{7}$ I proposed a possible resolution which I call "No-probability Interpretation of Probability".

The key idea of my proposal is this: there is no such property of an event as its "probability". That is why the standard interpretations fail to give a sound definition of 
probability; and that is why empirical sciences like physics can manage without such a definition. Whenever we use the term "probability" in scientific discourse, its meaning varies from context to context: it means different dimensionless $[0,1]$-valued physical quantities, or more precisely, different dimensionless normalized measures composed by different physical quantities in the various specific situations. Moreover, these context-dependent meanings reduce the concept of "probability" to ordinary physical quantities of empirical meanings, like relative frequency on a finite sample, ratio of phase-space volumes, or the quantities on the right hand side of formulas (1)-(2).

Consider my example in Point 6. One can calculate the average waiting time only from the fact that the trains come in every 5 minutes and that the moments of time when I enter to the station are uniformly distributed. These facts are ordinary, empirically verifiable, physical facts. The calculation requires only kinematics, without even mentioning "probabilities". If, however, someone would like to enforce a probabilistic language, the calculation of the average waiting time could be presented in the following way: I don't know when I entered to the station relative to the arrival of the train. Since all moments of time of my entering are of equal probability, I calculate with a uniform probability distribution... And the result will be the same. But, there appeared a term-probability — which has no definition. The statements containing this term, like "all moments of time of my entering to the station are of equal probability", are meaningless; it is impossible to verify whether they are true or not. As the example shows, however, the concept of "probability" is completely needless.

The research was partly supported by the OTKA Foundation, No. K 68043.

\section{Notes}

${ }^{1}$ H. Reichenbach, The Theory of Relativity and A Priori Knowledge. Berkeley and Los Angeles: University of California Press 1965.

${ }^{2}$ L. E. Szabó, "Empirical Foundation of Space and Time”, in M. Suárez, M. Dorato and M. Rédei (eds.), EPSA07: Launch of the European Philosophy of Science Association. Dordrecht: Springer 2009.

${ }^{3}$ J. Earman and W. Salmon, "The Confirmation of Scientific Hypotheses", in: M. H. Salmon, et al. (eds.), Introduction to Philosophy of Science. Englewood Cliffs, New Jersey: Prentice Hall 1992.

${ }^{4}$ A. Hájek, "Interpretations of Probability", in E. N. Zalta (ed.), The Stanford Encyclopedia of Philosophy (Summer 2003 Edition), http://plato.stanford.edu/archives/sum2003/entries/probability-interpret.

${ }^{5}$ D. Lewis, "A Subjectivist's Guide to Objective Chance", in: Philosophical Papers Volume II. New York: Oxford University Press 1987, p. 86.

${ }^{6}$ R. Frigg and C. Hoefer, "Determinism and Chance from a Humean Perspective", in this volume.

${ }^{7}$ L. E. Szabó, "Objective probability-like things with and without objective indeterminism", Studies in History and Philosophy of Modern Physics 38, 2007, pp. 626-634. 